\title{
e-Phaïstos
}

e-Phaïstos Revue d'histoire des techniques / Journal of the history of technology

VIII-2 | 2020

Aviation : émergence d'un complexe technique, 1900-1930

\section{Le Colegio Militar de Bogota (1848-1884). La mise en place d'un enseignement supérieur scientifique et technique après l'indépendance de la Colombie}

The Colegio Militar de Bogota (1848-1884). The Establishment of a Higher

Scientific and Technical Education in the Wake of the Independence of Colombia

\section{Bertrand Eychenne}

\section{(2) OpenEdition}

Journals

\section{Édition électronique}

URL : https://journals.openedition.org/ephaistos/7876

DOI : 10.4000/ephaistos.7876

ISSN : 2552-0741

Éditeur

IHMC - Institut d'histoire moderne et contemporaine (UMR 8066)

\section{Référence électronique}

Bertrand Eychenne, « Le Colegio Militar de Bogota (1848-1884). La mise en place d'un enseignement supérieur scientifique et technique après l'indépendance de la Colombie », e-Phaïstos [En ligne], VIII-2 I 2020, mis en ligne le 27 octobre 2020, consulté le 17 septembre 2021. URL : http://

journals.openedition.org/ephaistos/7876 ; DOI : https://doi.org/10.4000/ephaistos.7876

Ce document a été généré automatiquement le 17 septembre 2021.

Tous droits réservés 


\section{Le Colegio Militar de Bogota}

(1848-1884). La mise en place d'un enseignement supérieur scientifique et technique après l'indépendance de la Colombie

The Colegio Militar de Bogota (1848-1884). The Establishment of a Higher

Scientific and Technical Education in the Wake of the Independence of Colombia

\section{Bertrand Eychenne}

Thèse : références bibliographiques

Bertrand EYCHENNE, Le Colegio Militar de Bogota (1848-1884). La mise en place d'un enseignement supérieur scientifique et technique après l'indépendance de la Colombie. Thèse de doctorat en Histoire des sciences et des techniques. Université Paris-Saclay, soutenue le 9 juillet 2018, un volume de texte (640 p).

Directeur de thèse

Renaud d'ENFERT, professeur, Université de Picardie Jules Verne

Jury

Luis Carlos ARBODEL, professeur, Universidad del Valle, Cali

Bruno BELHOSTE, professeur, Université Paris 1 Panthéon-Sorbonne

Anne BIDOIS, maître de conférences, Université de Rouen

Virginie FONTENEAU, maître de conférences HDR, Université Paris-Sud, Paris-

Saclay

Nicolas HATZFELD, professeur, Université d'Évry-Val d'Essonne, Paris-Saclay

(Président)

Martina SCHIAVON, maître de conférences HDR, Université de Lorraine 
1 Le Colegio Militar (l'École militaire) est un établissement d'enseignement supérieur des sciences fondé à Bogota en 1848. Lorsque, l'année suivante, le président de la Nueva Granada (future Colombie) Tomas Cipriano de Mosquera demande aux députés d'accorder les crédits nécessaires au maintien de cette école, il leur rappelle que : «Cet établissement d'éducation n'est pas seulement un institut pour créer des officiers savants: c'est l'unique établissement de la République où peuvent se former des ingénieurs civils capables de prêter à la Nation des services importants et utiles ».

2 Mosquera attire ainsi l'attention des députés sur trois problèmes relatifs à la création du Colegio Militar :

La nécessité pour le pays de former, après son indépendance, des officiers militaires et des ingénieurs civils;

Mais aussi la volonté politique du pouvoir de soutenir cette formation considérée comme utile au développement matériel du pays ;

Et, enfin, la difficulté de mettre en place une telle formation scientifique et technique, que seul le Colegio Militar parvient à assurer.

3 Ces questions, sur lesquelles je reviendrai, se trouvent au cœur de l'étude de cette institution scolaire du Colegio Militar. Je l'ai menée dans une perspective d'histoire des sciences et de leur enseignement. Mais elle se situe également au croisement des histoires politique et sociale.

4 Mon attention a été attirée sur le Colegio Militar de Bogota en 2013 lorsque j'ai entamé des recherches dans le cadre d'un Master 2 en Histoire des Sciences et des Techniques, sous la direction de Marc Moyon. Installé à Bogota depuis 2009, cette reprise d'études m'a conduit à me tourner vers l'histoire des sciences en Colombie. Après avoir convenu avec mon directeur de master de consacrer mes recherches à un texte de mathématiques conçu dans ce pays, mon choix s'est arrêté sur les Leçons de géométrie analytique publiées en 1850 par Lino de Pombo, un professeur du Colegio Militar. Cet ouvrage m'était, en effet, accessible à la Bibliothèque nationale de Colombie et il présentait les particularités d'être l'un des plus anciens publiés par un auteur colombien mais aussi l'un des rares destinés à l'enseignement supérieur.

5 Cette première recherche m'a fait prendre consciences de l'importance du Colegio Militar pour l'enseignement des mathématiques en Colombie, car je découvrais que la plupart des textes parus dans le pays au $\mathrm{XIX}^{\mathrm{e}}$ siècle avaient été écrits par des enseignants de cette école. Les archives du Colegio Militar conservées aux Archives nationales de Colombie (Archivo General de la Nación) m'ont alors permis de retracer les conditions dans lesquelles le cours de Pombo avait été conçu durant les premières années de l'école.

6 Parallèlement, la consultation de l'historiographie sur l'enseignement des sciences en Colombie au XIX' siècle a conforté l'impression que j'avais du rôle joué par le Colegio Militar dans ce domaine. Pour les historiens des sciences tels que Clara Helena Sanchez, professeur à la Universidad Nacional de Bogota, le Colegio Militar a en effet fortement contribué au développement des études mathématiques d'un niveau supérieur et à la constitution d'une communauté mathématique (Sanchez-Bottero 2002 :240, Llull 1999 : 689). Par ailleurs, l'historien nord-américain Franck Safford affirme que, dans ce contexte difficile, le Colegio Militar parvint à former « une grande partie des ingénieurs qui travaillèrent à la construction des routes et des chemins de fer et qui enseignèrent 
les mathématiques et l'ingénierie durant la deuxième moitié du XIX siècle » (Safford 2014 : 341).

7 Pourtant, malgré cette place attribuée au Colegio Militar pour l'enseignement des sciences et des techniques, l'historiographie ne donnait de cette école qu'une vision partielle et imprécise, soit parce qu'elle n'abordait que certains aspects de l'établissement, soit parce qu'elle le considérait dans une perspective beaucoup plus vaste, telle que l'histoire de l'ingénierie de l'époque précolombienne à nos jours. Par ailleurs, ces travaux adoptaient le plus souvent un point de vue institutionnel, en se concentrant sur l'étude des textes officiels, sans parvenir à vérifier leurs conditions d'application.

8 La comparaison de ces travaux faisait aussi ressortir des divergences. Par exemple, sur la période durant laquelle l'école aurait fonctionné, sur les enseignants impliqués ou encore sur les modèles à partir desquels elle aurait été fondée. Mais j’ai rapidement réalisé que ces désaccords traduisaient surtout des difficultés inhérentes à l'étude du Colegio Militar. Face à cette institution, le chercheur se trouve en effet confronté aux changements de noms et de statuts de l'établissement (quatre en moins de quarante ans, avec une école qui dépend alternativement du secrétariat de la Guerre ou de l'Intérieur), à des périodes d'interruption, ou, encore, à la difficulté de rassembler des sources.

9 À ces questions d'ordre historiographique et méthodologique soulevées par mes premières recherches sur le Colegio Militar se sont ajoutées des problématiques que je commençais à découvrir comme, par exemple, la difficulté pour les dirigeants colombiens d'établir, après l'indépendance du pays en 1819 , un enseignement des sciences et une formation militaire.

10 Tout ceci m'a incité à centrer mon travail de doctorat sur cette institution. Il s'agissait d'étudier, comme je l'ai évoqué en introduction, les enjeux, dans ce contexte postcolonial, de la mise en place d'un établissement d'enseignement scientifique et technique de niveau supérieur; d'observer les solutions apportées par le pouvoir politique aux multiples obstacles qui s'opposaient à cette création; d'établir une méthodologie me permettant d'analyser cette école tout en la considérant dans une perspective plus large que la seule transmission de connaissances.

11 Ce dernier point m'a conduit à employer les termes d'« institution éducative » afin de désigner une structure dont l'objectif est de former des individus en développant leurs connaissances, mais aussi en leur permettant d'acquérir des valeurs et des comportements. Je souhaitais également apporter des éclairages plus précis que ceux fournis par l'historiographie existante sur la manière dont le Colegio Militar avait pu contribuer au développement d'un enseignement supérieur des sciences et des techniques en Colombie.

12 Cette notion d'enseignement supérieur, qui apparaît au XIX siècle, demande d'ailleurs à être précisée dans son contexte local et historique. Aussi appelé par les textes officiels «enseignement professionnel », elle désigne, le plus souvent, les études débouchant sur des diplômes qui, depuis l'époque coloniale sont, en Colombie, le monopole des universités, notamment pour les carrières traditionnelles du droit, de la médecine et de la théologie. Cette question des diplômes remis par l'institution renvoyait également à la professionnalisation des ingénieurs civils colombiens et à celle des enseignants de sciences. 

un patient travail de collecte et de dépouillement. Pour les périodes où l'école est sous la tutelle du secrétariat de la Guerre, les documents sont conservés aux Archives Nationales de Colombie où, en l'absence de catalogue informatisé, il m'a fallu épuiser les index imprimés pour rassembler près de 5000 documents relatifs à l'école. Pour la période où elle dépend de l'université, une partie des documents est malheureusement perdue. Les quelques centaines qui nous sont parvenus figurent aux archives de la Universidad Nacional et sont d'un accès plus facile. connaissance du contexte politique, social et éducatif du XIX ${ }^{\mathrm{e}}$ siècle colombien. Pour appréhender ce dernier, j'ai réuni les textes législatifs et réglementaires de l'instruction publique adoptés entre l'indépendance du pays et les années 1880 puis je les ai rapprochés des rapports annuels de plusieurs secrétaires d'État qui donnaient une vision plus claire du contexte politique.

Ces éléments ont été complétés par des sources secondaires qui, dans le domaine de l'histoire de l'enseignement en Colombie, ont tendance à se concentrer sur certaines périodes, comme la réforme de l'enseignement primaire adoptée par le gouvernement libéral radical dans les années 1870. Il est donc parfois difficile d'acquérir une vision précise et continue de l'évolution de l'enseignement secondaire au XIX siècle. Le dépouillement et l'analyse de ces sources, comme la nécessité de prendre en compte le contexte éducatif, politique et social m'ont amené à m'interroger sur l'approche méthodologique que je devais adopter pour entreprendre l'étude d'une institution éducative.

16 En effet, d'une part le Colegio Militar possède de multiples facettes : c'est un lieu de transmission de savoirs scientifiques et techniques, mais aussi un lieu de formation d'ingénieurs civils et de cadres de l'armée, un lieu d'acquisition de certains habitus, un lieu d'expression du pouvoir et des politiques publiques. D'autre part, une telle étude renvoie à divers domaines de la société. Le Colegio Militar intervient en effet dans des champs qu'il contribue à réguler comme, par exemple, l'enseignement supérieur ou la formation militaire. J'ai alors entrepris de constituer un environnement intellectuel pouvant m'aider à m'orienter et à faire des choix. J'ai ainsi établi un corpus de travaux historiques sur des institutions du même type, aussi bien en Europe, qu'en Amérique du Nord et en Amérique Latine.

17 Parmi ces travaux, j'ai tout d'abord été guidé par l'étude de Bruno Belhoste sur l'École polytechnique (Belhoste 2003), à la fois pour la méthodologie suivie comme pour la place tenue historiquement par cette école dans le domaine de la formation scientifique. D'autres monographies ont alimenté mon questionnement méthodologique, comme celle d'Anne-Françoise Garçon (Garçon 2004) sur l'École des mines de Saint-Étienne, ou encore les études de l'École centrale des arts et manufactures de Paris de John Hubbel Weiss (Weiss 1982) et celle des écoles des arts et métiers par Charles Day (Day 1991). Elles concernent des établissements orientés vers la formation technique et l'industrie et présentent des exemples d'études des populations d'élèves dont j'ai pu m'inspirer. Concernant l'Espagne, je me suis nourri de divers travaux sur les Académies militaires espagnoles, notamment ceux Helena Ausejo (Ausejo, Velamazan 1989) et pour les États-Unis, des études sur l'académie de West Point (Crackel 2002) et le Rensselaer Polytechnic Institute (Rickett 1934). Enfin, j'ai tenu à associer à ce corpus des établissements de formation scientifique d'Amérique Latine, 
comme la Academia de matemáticas de Caracas qui présente plusieurs points communs avec le Colegio Militar.

Ce corpus de travaux sur des établissements dédiés à la formation d'ingénieurs civils ou militaires a permis de préciser ma démarche méthodologique. Ils m'ont en effet présenté un éventail d'approches qui m'a aidé à dégager les principaux domaines sur lesquels je pouvais articuler mon étude du Colegio Militar à savoir: sa structure, sa fonction et la culture transmise. Par ailleurs, ces études font apparaître des spécificités de chaque établissement. Elles ont ainsi attiré mon attention sur la nécessité d'affiner ma démarche afin de faire émerger des caractéristiques du Colegio Militar. J'ai alors pris le parti d'écarter un plan chronologique pour placer au cœur de mon travail l'analyse des différents aspects de cette institution éducative qu'est le Colegio Militar. J'ai ainsi décomposé mon étude en trois parties thématiques abordant successivement l'aspect institutionnel, les enseignements et enfin les élèves.

La première partie de ma thèse est donc consacrée aux aspects institutionnels. Elle étudie les liens entre l'école et le pouvoir politique. Cette approche découle de la nécessité d'ouvrir, à partir de cet objet d'étude bien délimité, le champ d'investigation à l'histoire politique et économique du pays.

Il m'a paru essentiel d'insister sur la place donnée à l'éducation dans le processus postcolonial de construction de l'État. Elle intervient ainsi dans la définition des partis politiques colombiens dont les luttes de pouvoir influent sur la création et le devenir du Colegio Militar. De plus, face à l'agitation du siècle (le pays connaît en effet pas moins de cinq guerres civiles entre 1848 et 1884) et aux bouleversements que subit le Colegio Militar, il m'a semblé que la prise en compte du contexte politique pouvait faire apparaître des continuités ou des caractéristiques de l'école. Elle permettait également de suivre l'évolution des objectifs qui lui sont assignés. En effet, les textes réglementaires insistent parfois sur certains aspects de l'établissement dont l'importance peut être mieux comprise lorsqu'on les fait entrer dans les logiques des programmes politiques des différents gouvernements.

21 Par exemple, l'intégration de l'école à la Universidad Nacional en 1868, sous le nom d'Escuela de Ingeniería (École d'ingénieurs), est directement lié à l'accession au pouvoir du parti libéral radical et à la mise en place d'un système fédéral. En effet, la création de l'université s'inscrit dans une politique de contrôle et de centralisation de l'enseignement supérieur sur lequel le pouvoir exécutif compte pour renforcer les liens entre les États de l'union. Le Colegio Militar participe à ce dispositif, notamment en accueillant des élèves boursiers de tous ces États.

22 La deuxième partie de ma thèse porte sur la transmission des savoirs par le Colegio Militar. Pour cette étude, je me suis référé à la notion de curriculum telles qu'elle fut développée dans les années 1970 par la " Nouvelle sociologie de l'éducation anglaise » menée par Basil Bernstein et Michael Young. J'envisage ainsi le curriculum comme un parcours éducatif composé d'expériences d'apprentissage diverses réalisées dans le cadre d'une institution.

23 Le Colegio Militar étant une école d'enseignement scientifique et technique, j'ai insisté particulièrement sur le rôle central tenu par les mathématiques et le dessin qui apparaissent comme des disciplines structurantes. Plus précisément, j'ai pu indiquer comment la méthode de l'analyse mathématique consistant à décomposer un problème en éléments simples est mise en avant comme une méthode générale de résolution de 
problèmes dans divers enseignements tels que la géométrie analytique, le calcul différentiel ou la géométrie descriptive.

Enfin, la dernière partie de cette étude est consacrée aux élèves du Colegio Militar. Elle décrit la composition de cette population et aborde des aspects de l'école en marge des contenus enseignés comme le régime disciplinaire ou la transmission de valeurs. J'ai également voulu interroger l'impact de l'école sur la formation des ingénieurs civils qu'évoque l'historien Franck Safford, en suivant le devenir des élèves issus du Colegio Militar. J'observe ainsi, à partir des années 1870, l'émergence d'un groupe professionnel qui se met en place, à l'instigation d'anciens élèves du Colegio Militar, par la création d'une société d'ingénieurs nationaux (la Sociedad Colombiana de Ingenieros). Cette société, à mi-chemin entre la corporation et la société savante, vise une légitimité professionnelle et une reconnaissance institutionnelle lui permettant d'exercer un contrôle de la profession d'ingénieur civil. Sa démarche s'appuie notamment sur la mise en avant d'un savoir scientifique et technique en adéquation avec le contexte géographique local.

Comme je l'ai indiqué, la comparaison d'études des différentes écoles et la prise en compte du contexte politique m'ont permis de faire ressortir des caractéristiques de l'école. Parmi ces éléments, j'aimerais insister, tout d'abord, sur la mise en place d'une formation mixte à la fois militaire et civile. Si je me base sur les études rassemblées dans mon corpus, cette caractéristique est assez rare et semble par conséquent présenter une certaine singularité. De plus, la composante militaire a souvent été écartée ou minimisée par l'historiographie. Pourtant, j'ai pu montrer que si la formation civile domine sur l'ensemble de la période, l'aspect militaire ne joue pas moins un rôle essentiel à la fois lors la création du Colegio Militar et durant la dernière période postérieure à 1880 .

Ainsi, comme cela apparaît très clairement dans les projets de loi précédant sa création, l'école doit sa fondation à l'insistance des Secrétaires de la Guerre successifs sur la nécessité pour l'État d'assurer la formation des officiers de l'armée. Celle des ingénieurs civils n'est en fait ajoutée que lors de la réécriture du texte de loi par les sénateurs, afin d'élargir l'apport de cette école soutenue par les finances publiques.

Cette formation à la fois militaire et civile est par la suite maintenue dans les textes officiels. Son principe fait consensus auprès des différents gouvernements et apparaît comme une continuité. Pourtant, les dirigeants, en l'intégrant à leurs programmes, en donnent des interprétations différentes. Tantôt, elle s'inscrit dans une politique sécuritaire, visant à renforcer la défense nationale, tantôt elle répond à un projet de démilitarisation cherchant à remplacer l'armée permanente par des milices. Des inflexions apparaissent alors dans les proportions consacrées aux versants militaires et civils de la formation du Colegio Militar. Ces ajustements montrent en retour comment les objectifs de l'établissement peuvent être adoptés et adaptés par les différents gouvernements qui maintiennent ainsi un soutien de l'État indispensable au financement de l'école.

Revenons sur la notion de curriculum. Elle permet de porter un regard large sur les apprentissages, en distinguant notamment ceux définis par les programmes officiels que l'on peut désigner comme le curriculum formel, et ceux mis en place dans l'établissement, c'est-à-dire, le curriculum réel. Ce dernier comprend aussi bien les notions acquises par les élèves que les valeurs et les comportements transmis par cette partie non institutionnelle du curriculum appelée le curriculum caché. Le curriculum 
permet donc d'aborder les apprentissages de manière globale et cohérente, en considérant les connaissances, mais aussi les savoir-faire et les savoir-être. Par ailleurs, il permet de penser à la fois le contenu et la forme des apprentissages dispensés par une institution éducative. Il est alors possible d'envisager la fabrication du savoir lié à l'enseignement comme une action de terrain, c'est-à-dire en relation avec l'institution, l'action des professeurs, les méthodes, l'évaluation et les conditions matérielles d'enseignement. Enfin, sur un plan plus social, le curriculum apporte des éclairages sur les relations entre les différents acteurs car, comme l'affirme Basil Bernstein, la sélection et l'organisation des savoirs «reflète(nt) une distribution du pouvoir et les principes du contrôle social » (Bernestein 1975).

Tous ces éléments sont mobilisés lors de l'étude l'évolution du curriculum du Colegio Militar qui, entre 1848 et 1884, passe de 4 à 7 années. Cette question permet notamment de montrer qu'en dehors des textes officiels, certains acteurs tels que les administrateurs de l'école, les enseignants ou même les élèves peuvent intervenir sur celui-ci. On observe ainsi certains moments forts de l'évolution du curriculum. Il se construit tout d'abord, durant les premières années, autour des mathématiques, du dessin et de la législation militaire. Mais les archives de l'école montrent des rapports de force et des rivalités entre les enseignants sur l'importance à accorder à chaque matière. Ce désaccord se résout finalement avec la réduction de l'enseignement de la législation militaire au profit d'un renforcement des mathématiques.

Lorsque, dans les années 1860, le Colegio Militar est intégré à la Universidad Nacional, le programme initial de l'école est, dans un premier temps, consolidé. Les professeurs impliqués ont été pour la plupart formés par l'école elle-même durant la période précédente. Ils reprennent l'enseignement qu'ils ont suivi comme élève, mais certains d'entre eux ajoutent des variations en intégrant des éléments de leur expérience professionnelle. C'est le cas, par exemple, du cours de géométrie pratique pour lequel le professeur Manuel $\mathrm{H}$. Peña s'appuie sur ses travaux comme géomètre-arpenteur pour apporter aux élèves des notions adaptées à la géographie locale. Profitant de la concentration des ressources matérielles et humaines que permet l'université, les enseignants interviennent également pour obtenir l'extension du curriculum aux matières expérimentales. Ce sont ensuite les élèves qui obtiennent la correction de certaines incohérences. Le curriculum connaît également progressivement un développement des enseignements spécifiques et appliqués mais, avec le renforcement des enseignements militaires dans les 1880 , le gouvernement doit finalement envisager la séparation de l'école en deux établissements distincts, l'un civil, l'autre militaire.

31 L'étude d'une institution éducative dans un pays sud-américain tel que la Colombie offre l'opportunité d'un décentrement du regard et invite à s'interroger sur les mécanismes des transferts de savoirs. Ces questions ont parfois été envisagées par l'historiographie qui a avancé l'hypothèse de modèles d'écoles étrangères adoptés pour la fondation du Colegio Militar. On a pu évoquer ainsi l'École polytechnique ou encore l'académie de West Point. Ces pistes du transfert m'ont dans un premier temps poussé à rechercher dans les sources primaires des références précises à d'éventuels modèles extérieurs. J'ai parallèlement entrepris de comparer le Colegio Militar avec d'autres établissements ayant pu influencer ses concepteurs et j'ai rassemblé des informations sur les circulations de connaissances vers mais aussi à l'intérieur de la Colombie, au XIX ${ }^{\mathrm{e}}$ siècle. 

être une préoccupation des dirigeants colombiens dès l'accession du pays à l'indépendance. Mais elle n'est pas l'apanage des États situés sur ce que l'on appelle parfois la "périphérie ». Elle apparaît en effet également dans des pays d'Europe ou d'Amérique de Nord, comme le montre Damiano Matasci (Matasci 2015). De plus, cette recherche par les élites locales de modèles extérieurs prend souvent la forme d'une démarche qui ne s'arrête pas à une copie conforme mais vise plutôt, comme l'affirme Victor Cousin en 1832, « à emprunter partout ce qui est bien, et à le perfectionner en se l'appropriant » (Cousin 1832: 396). Il s'agit donc pour les dirigeants de s'inspirer de sources diverses, d'y puiser des éléments précis, sélectionnés, et de les adapter au contexte local comme aux objectifs visés.

Santander, visitent dans les années 1830 diverses institutions scolaires d'Europe et des États-Unis. Des diplomates sont également chargés de transmettre au gouvernement des rapports sur des établissements implantés dans les pays où ils sont en poste. Cette démarche ne m'est pas apparue comme une recherche d'un modèle spécifique à transposer. Elle semble plutôt correspondre à une quête de légitimité poursuivie par les dirigeants par rapport à leur politique éducative. Elle présente en effet des similitudes avec les références européennes utilisées dans les discours politiques qu'analyse Frédéric Martinez (Martinez 2001).

Par ailleurs, se pose la question des circulations des connaissances vers et à partir du Colegio Militar à différents niveaux: sur le plan institutionnel, comme je viens de l'évoquer, mais aussi au niveau de l'élaboration du curriculum. Ainsi, j'ai pu montrer qu'à l'ouverture de l'établissement, la mise en place du programme d'études repose sur des enseignants étrangers ou des Colombiens formés dans d'autres pays. Parmi eux figurent, outre Lino de Pombo, Colombien formé en Espagne, le Français Aimé Bergeron dont j'ai retracé le parcours qui l'a conduit du sud de la France à la Nueva Granada. Pour appréhender ces circulations, j'ai également tenté de mettre en évidence certaines sources utilisées par des enseignants du Colegio Militar, notamment des sources françaises comme le cours de géométrie élémentaire (1837) de Vincent ou celui d'analyse de Sturm (1857) (Vincent 1837, Strum 1857), mais aussi de montrer leur diversité. Selon les enseignements, ces textes sont utilisés de différentes manières : comme manuels de la classe ou sous forme d'extraits sélectionnés par le professeur, alors que d'autres, comme Pombo, préfèrent utiliser plusieurs sources qu'ils adaptent et s'approprient pour concevoir leur propre cours.

35 Enfin, il convient de souligner le rôle joué par les publications des enseignants du Colegio Militar dans la diffusion de connaissances scientifiques sur le plan national à partir de l'établissement. Certains de ces manuels sont utilisés par l'école elle-même, mais aussi par d'autres institutions et connaissent parfois une longévité remarquable grâce aux nombreuses rééditions. Ces publications prolongent ainsi l'enseignement établi à l'origine pour le Colegio Militar et étendent son public dans le temps comme dans l'espace vers ce qu'Annie Bruter appelle un «public différé » (Bruter 2008). La participation d'enseignants du Colegio Militar à d'autres institutions contribue également à cette diffusion des connaissances au niveau local.

Tous ces éléments soulignent qu'autour du Colegio Militar, sur le plan institutionnel comme au niveau des cours établis par les enseignants, les circulations de connaissances ne se font pas de manière linéaire selon un modèle que l'on chercherait à 
transposer intégralement en Colombie. Les transferts me semblent plutôt, dans le cadre de cette étude, se rapprocher de mécanismes décrits par Liliane Hilaire-Pérez et Catherine Verna (Hilaire-Pérez, Verna 2009) pour la circulation des savoirs techniques. Ils s'avèrent multiples mais limités, faisant intervenir des sources diverses et des acteurs variés, et parmi ces derniers, il convient de souligner le rôle des enseignants dont les actions individuelles liées à la conception des cours magistraux contribuent à cette hétérogénéité.

Ce travail me conduit à envisager certains prolongements. Je voudrais, pour conclure, en évoquer deux brièvement.

Tout d'abord, mes recherches m'ont fait prendre conscience de l'intérêt qu'il y aurait à établir une cartographie des établissements scolaires de Colombie au cours du XIX ${ }^{\mathrm{e}}$ siècle et de suivre leur évolution en commençant par la capitale Bogota. Ceci permettrait de dresser les contours du champ éducatif et d'évaluer la position de chaque institution dans celui-ci, ainsi que les relations établies entre elles. Il en découlerait une meilleure connaissance de l'offre locale d'enseignement scientifique et technique et notamment des circulations de personnes et de connaissances comme des situations de concurrence et de complémentarité qu'elle peut induire.

Par ailleurs, l'étude d'établissements scientifiques du même type que le Colegio Militar à travers l'Amérique Latine, où la plupart des pays connaissent des histoires similaires au $\mathrm{XIX}^{\mathrm{e}}$ siècle, permettrait de vérifier si le modèle que je dégage du Colegio Militar à savoir d'une école d'État apportant un enseignement militaire et civil, un cursus intégré et une sélection limitée, peut s'appliquer à d'autres institutions éducatives de la zone. Elle contribuerait également à prolonger une étude des circulations des savoirs à l'échelle du continent que j'ai seulement ébauchée, mais qui me semble constituer une perspective de recherche prometteuse.

\section{BIBLIOGRAPHIE}

AUSEJO Elena, VELAMAZAN Angeles, « Los planes de estudio en la Academia de Ingenieros del Ejercito de España en el siglo XIX », Llull, vol.12, décembre 1989, p.415-453

BELHOSTE Bruno, La formation d'une technocratie, l'École polytechnique et ses élèves de la Révolution au Second Empire, Paris, Belin, 2003

BERNESTEIN Basil, « On the Classification and Framing of Educational Knowledge », YOUNG

Michael, Knowledge and Control, Londres, Cassell \& Collier Macmillian, $4^{\mathrm{e}}$ ed., 1976

BRUTER Annie, «Le cours magistral comme objet d'histoire ", Histoire de l'éducation, n¹20, septembre 2008, p.5-32

COUSIN Victor, Rapport sur l'état de l'instruction publique dans quelques pays d'Allemagne, et particulièrement en Prusse, Paris, Imprimerie Royale, 1832

CRACKEL Theodore J., West Point: A bicentennial history, Lawrence, University Press of Kansas, 2002 DAY Charles R., Les Écoles d'Arts et Métiers, Paris, Belin, 1991 
GARÇON Anne-Françoise, Entre l'État et l'usine. L'École des mines de Saint-Étienne au XIXe siècle, Rennes, Presse Universitaires de Rennes, 2004

HILAIRE-PÉREZ Liliane, VERNA Catherine, « La circulation des savoirs techniques du Moyen-âge à l'époque moderne. Nouvelles approches et enjeux méthodologiques », Tracés, Revue de Sciences humaines, $\mathrm{n}^{\circ} 16,2009$, p.25-61

MARTINEZ Frédéric, El nacionalismo cosmopolita, la referencia europea en la construcción nacional en Colombia, 1845-1900, Institut français d'études andines, Bogota, 2001

MATASCI Damiano, L'école républicaine et l'étranger, Paris, ENS éditions, 2015

RICKETT Palmer C., History of Rensselaer Polytechnic Institute 1824-1914, New York, John Wiley and sons, $3^{\mathrm{e}}$ ed., 1934

SAFFORD Frank, El ideal de lo practico, El desafío de formar una élite técnica y empresarial en Colombia, Medellin, Fondo editorial Universidad EAFIT, $2^{\mathrm{e}}$ ed., 2014

SANCHEZ BOTERO Clara Helena, « Cien años de historia de la matemática en Colombia 1848-1948 ", Revista de la Academia Colombiana de Ciencias, vol.26, n99, juin 2002

STURM Charles, Cours d'analyse de l'École polytechnique, Paris, Maillet-Bachelier, 1857

VINCENT Alexandre-Joseph-Hidulphe, Précis de géométrie élémentaire, Paris, Bachelier, 1837

WEISS John Hubbel, The Making of Technological Man, Cambridge, MIT Press, 1982

\section{RÉSUMÉS}

Lorsque la Colombie s'émancipe de la Couronne d'Espagne en 1819, elle doit consolider son indépendance et faire face à divers obstacles qui s'opposent à l'instauration d'un enseignement scientifique et technique stable. En prenant en compte ce contexte postcolonial et en se concentrant sur le Colegio Militar de Bogota, une école d'ingénieurs civils et militaires, entre 1848 et 1884 , ce travail retrace le processus ayant conduit à la mise en place d'un tel enseignement et suit son évolution au cours de la deuxième moitié du XIX ${ }^{e}$ siècle. L'influence du pouvoir s'avère alors constante et fait ressortir des caractéristiques de l'établissement telles que l'enseignement mixte, militaire et civil, la recherche d'utilité ou l'absence de sélection. Par ailleurs l'étude du curriculum du Colegio Militar indique dans quelle mesure la vision institutionnelle influencée par les idéologies des partis transparait dans les enseignements. Elle fait apparaître également la capacité des différents acteurs à modifier à leur niveau ce curriculum et parvient à suivre son histoire dans sa continuité, malgré l'hétérogénéité de ces interventions. L'analyse des notions scientifiques et techniques transmises par ce curriculum, montre également comment, en s'adaptant au contexte, la mise en place d'une diffusion des savoirs s'accompagne d'une production scientifique. Le décentrement du regard qu'implique cette étude, nous invite à aborder la question du transfert de connaissances en montrant comment l'analyse d'une institution éducative permet d'appréhender ces mécanismes dans leur complexité. Ces circulations seront considérées à différentes échelles au niveau local en lien avec la fonction régulatrice de l'établissement dans le champ éducatif, au niveau du continent sud-américain et vers les centres de production scientifiques d'Europe et d'Amérique du Nord. Enfin, ce travail permet d'apporter des éclairages sur la constitution et l'émergence en Colombie d'un groupe professionnel - les ingénieurs civils - étroitement lié à celui des enseignants de sciences. En suivant le parcours des anciens élèves du Colegio Militar, il révèle les stratégies adoptées par ce groupe social pour faire reconnaître à la fois sa profession et sa légitimité à l'exercer. 
As Colombia becomes emancipated from the Spanish Crown in 1819, it has to strengthen its independence and is thus faced with a number of obstacles which come in the way of a stable scientific and technical education. By taking into account this postcolonial context and by focusing on the Colegio Militar of Bogota, a school of civil and military engineering between 1848 and 1884 , this study describes the process which led to the foundation of this kind of teachings and follows its evolution during the second half of the 19th century. The influence of power proves to be constant at the time and brings out such specificities as its mixed education curriculum, military and civil, its quest for utility or the absence of a selection process. Furthermore, the study of its curriculum reveals how its institutional vision influenced by party ideology shows up through the teachings. It also demonstrates the ability of the various players to alter, at their level, this curriculum and manages to establish the continuity of its history despite the heterogeneity of their actions. Similarly, the analysis of the scientific and technical notions conveyed by this curriculum illustrates how, by adapting to the context, the diffusion of knowledge comes with scientific production. The decentralization entailed by our study leads us to consider the issue of transfer of knowledge, by showing how the study of an educational institution allows to tackle these mechanisms in their complexity. These circulations will be considered on different levels, locally with the regulating function of the school in the educational field, and largely within the South American continent and toward the main hubs of scientific production in Europe and North America. Finally, this study sheds some light on the constitution and emergence of a professional group in Colombia namely civil engineers, which is tightly linked to the science teachers group. By following the trail of former students of the Colegio Militar, we become acquainted with the strategies they adopt to ensure that both their profession and the legitimty to practise is acknowledged.

\section{INDEX}

Mots-clés : histoire des techniques, histoire des sciences, enseignement technique, ingénieur, circulation des savoirs, culture scientifique et technique

Thèmes : Positions de thèse

Keywords : history of technology, history of science, technical education, engineering, circulation of knowledge

\section{AUTEUR}

\section{BERTRAND EYCHENNE}

Docteur en histoire des sciences et des techniques, Bertrand Eychenne est chercheur associé au Groupe d'Histoire et de Diffusion des sciences d'Orsay (GHDSO), EA 1610 de l'Université ParisSaclay. Ses travaux de recherche en cours portent sur l'étude historique des institutions scolaires et l'histoire des formations scientifiques et techniques. Il travaille en particulier sur la formation des ingénieurs et sur l'enseignement apporté aux ouvriers et aux apprentis. 